\title{
Derivation of the Euler Equations from a Caricature of Coulomb Interaction
}

\author{
Yann Brenier
}

Vienna, Preprint ESI 702 (1999)

May 20, 1999

Supported by Federal Ministry of Science and Transport, Austria Available via http://www.esi.ac.at 


\title{
DERIVATION OF THE EULER EQUATIONS FROM A CARICATURE OF COULOMB INTERACTION
}

\author{
Yann Brenier
}

\begin{abstract}
A caricature of collisionless plasma involving $2 N$ particles of opposite charge is introduced. The $N$ first particles are called 'ions' and don't move. The $N$ other particles are called 'electrons'. At each time, there is a one-to-one matching between electrons and ions and each pair is linked by a 'spring' so that each electron oscillates with fixed frequency $\epsilon^{-1}$. The essential point is that the matching between electrons and ions is updated at every discrete time $n \tau, n=0,1,2, \ldots$, so that the total potential energy of the system stays minimal. This leads to a non trivial interaction which turns out to be a caricature of Coulomb interaction. It is proven that, provided the $N$ ions are equally spaced in a bounded domain $D$ and $\epsilon, \tau$ and $N^{-1}$ tend to zero at appropriate rates, the electrons behave as the fluid parcels of an incompressible inviscid liquid moving inside $D$ according to the Euler equations. Our proof relies on a result of P.Lax on the approximation of volume-preserving transformations by permutations.
\end{abstract}

\section{AMS classification 35Q35-76C (82C22)}

Keywords : particles, permutations, Coulomb interaction, Plasma Physics, Fluid Mechanics, Euler equation, Poisson and Monge-Ampère equations.

\footnotetext{
*Institut Universitaire de France, et Laboratoire d'analyse numérique, Université Paris 6, France, brenier@ann.jussieu.fr
} 


\section{Description of the dynamical system}

Consider a smooth compact domain $D$ in $\mathbf{R}^{d}$ with unit volume and set $N$ particles inside $D$. These particles are called 'ions' and their positions $A_{1}, \ldots, A_{N} \in D$ are supposed to be fixed. Now, consider $N$ other particles, called 'electrons', moving in the Euclidean space $\mathbf{R}^{d}$, with label $\alpha=1, \ldots, N$ and position $X_{\alpha}(t) \in \mathbf{R}^{d}$ at time t. A time step $\tau>0$ is fixed. In each time interval $n \tau \leq t<(n+1) \tau$, there is a one-to-one pairing $\alpha \rightarrow \sigma_{\alpha}$ between each electron $X_{\alpha}$ and a corresponding ion $A_{\sigma_{\alpha}}$. A spring links each pair so that each electron oscillates around the corresponding ion with fixed frequency $\epsilon^{-1}$ :

$$
\epsilon^{2} X_{\alpha}^{\prime \prime}+X_{\alpha}=A_{\sigma_{\alpha}}
$$

Of course, during the time interval $n \tau<t<(n+1) \tau$, the total energy

$$
E(t)=\frac{1}{2}\left\|X^{\prime}(t)\right\|^{2}+\frac{1}{2 \epsilon^{2}}\|X(t)-\sigma A\|^{2},
$$

is preserved. (In this equation, the following notations have been used :

$$
\begin{gathered}
X(t)=\left(X_{\alpha}(t)\right)_{\alpha=1}^{N}, \quad A=\left(A_{\alpha}\right)_{\alpha=1}^{N}, \\
\|Y\|^{2}=\frac{1}{N} \sum_{\alpha}\left|Y_{\alpha}\right|^{2}, \quad \sigma Y=\left(Y_{\sigma_{\alpha}}\right)_{\alpha=1}^{N},
\end{gathered}
$$

for all $Y=\left(Y_{\alpha}\right)_{\alpha=1}^{N} \in\left(\mathbf{R}^{d}\right)^{N}$.)

At each discrete time $t=n \tau$, the pairing is subject to change and $\sigma$ is updated to keep minimal the potential energy, namely

$$
\frac{1}{2 \epsilon^{2}}\|X(n \tau)-\sigma A\|^{2}
$$

among all permutations. (Notice that they may be several solutions, in which case we arbitrarily choose one of them.) So, $\sigma$ is time dependent, piecewise constant, and denoted by $\sigma(t)$. Of course, we assume both positions and velocities of each particle to be continuous at each discrete time $n \tau$ and we prescribe their values at time 0 . This gives a complete description of the dynamical system. Notice that the total energy, defined by (2), is preserved on each interval $n \tau<t<(n+1) \tau$, and can only decay at each time $n \tau$, by definition of $\sigma(n \tau)$. So the total energy is a non-increasing function of time. The possible dissipation is due to the fact that $\sigma(t)$ is updated only at $t=n \tau$, and not continuously in time (in which case, the system would be formally conservative). We have chosen to introduce the time step 
$\tau$ in order to have a non ambiguous definition of the dynamical system and also to get a system that can be exactly integrated on a computer, without further approximation.

\section{Derivation of the Euler equations}

The motion of an incompressible inviscid liquid moving inside $D$ is classically described by the Euler equations (see $[\mathrm{AK}],[\mathrm{MP}]$, for example)

$$
\partial_{t} v+(v \cdot \nabla) v+\nabla p=0, \quad \nabla \cdot v=0,
$$

where $p=p(t, x) \in \mathbf{R}$ is the pressure field, $v=v(t, x) \in \mathbf{R}^{d}$ is the velocity field, which is supposed to be parallel to the boundary $\partial D$, so that there is no material flux across the boundary. If the initial value $v(0, x) \in \mathbf{R}^{d}$ is smooth, then the Euler equations have a unique smooth solution, which is globally defined in time if $d=2$ and locally if $d=3$. Let us consider such a solution and fix a time interval $[0, T]$ on which $v$ is well defined and denote by $C$ any constant depending only on $D, T, v$ and $p$.

Theorem 2.1 Assume that $D$ can be split into $N$ disjoint subdomains $D_{\alpha}$ of equal volume and diameter not larger than $C h$, where $h=N^{-1 / d}$, each of them containing one and only one ion $A_{\alpha}$. Assume that the initial positions and velocities of the particles are given by

$$
X_{\alpha}(t=0)=A_{\alpha}, \quad X_{\alpha}^{\prime}(t=0)=v\left(t=0, A_{\alpha}\right),
$$

for $\alpha=1, \ldots, N$. Scale the parameters $\epsilon, \tau$ and $h=N^{-1 / d}$ so that

$$
h \leq C \epsilon^{8}, \quad \tau \leq C \epsilon^{4}
$$

Then

$$
\frac{1}{N} \sum_{\alpha}\left|X_{\alpha}^{\prime}(t)-v\left(t, X_{\alpha}(t)\right)\right|^{2} \leq C \epsilon^{2}
$$

Before giving the proof, we provide in the next section a geometrical interpretation of the theorem and we explain why the interaction of the particles can be seen as a caricature of Coulomb interaction. Of course, the reader only interested in the proof of the derivation of the Euler equations may go directly to section 4 . 


\section{Geometric and physical interpretations}

\subsection{A geometric approximation to the Euler equations}

Let us recall that the Euler equations, describing the motion of an inviscid incompressible fluid moving inside a smooth bounded domain $D$ of the Euclidean space $\mathbf{R}^{d}$, have a nice geometric interpretation, for which we refer to [AK]. They describe the geodesics on the group $G$ of all volume-preserving diffeomorphisms of $D$, where length are measured in the $L^{2}$ sense, $G$ being viewed as a subset of the Hilbert space $H=L^{2}\left(D, \mathbf{R}^{d}\right)$. Of course, this correspondence is somewhat formal and a lot of analytical difficulties are left behind [Sh], one of them, for example, being that, for all $d \geq 2$, the $L^{2}$ closure of $G$ is the (much larger) semi-group $S$ of all Borel Lebesgue-measure preserving map from $D$ into itself.

So, it is natural to look for either generalized ([Sh], [Br2]) or approximate geodesics. A simple way to define approximate geodesics is to consider the formal dynamical system

$$
X^{\prime \prime}+\nabla_{X}\left(\frac{d_{H}^{2}(X, G)}{2 \epsilon^{2}}\right)=0,
$$

in the configuration space $H$, where $\nabla_{X}$ is the functional gradient in $H$ and the potential involves the distance in $H$ between $X$ and $G$ (or, equivalently, between $X$ and $S$, the $L^{2}$ closure of $G$ ), namely :

$$
d_{H}(X, G)=\inf _{g \in G}\|X-g\|_{H}=\inf _{g \in S}\|X-g\|_{H}=d_{H}(X, S),
$$

where $\|\cdot\|_{H}$ is the Hilbert norm of $H$. (This approach is similar-but not identical- to Ebin's slightly incompressible flow theory [Eb], see also [RU] for finite dimensional mechanical systems.) To get an approximate finitedimensional Hamiltonian system, we set $N$ points $A_{1}, \ldots, A_{N}$ equally spaced in $D$, we substitute for $H$ and $G$ respectively $\left(\mathbf{R}^{d}\right)^{N}$ and the finite group of all permutations of the $A_{\alpha}$

$$
\left\{\left(A_{\sigma_{1}}, \ldots, A_{\sigma_{N}}\right) \in\left(\mathbf{R}^{d}\right)^{N}\right\},
$$

where $\sigma$ is any permutation of $\{1, \ldots, N\}$, and we keep unchanged (8), (9). Finally, a more tractable dynamical system is obtained by introducing a time step $\tau$ and updating the potential energy only at discrete times $n \tau$. This exactly leads back to our system of particles and it is no longer surprising that the Euler equations can be recovered as $\epsilon, n^{-1}$ and $\tau$ go to zero. 


\subsection{A caricature of Coulomb interaction}

In this subsection, we give a formal argument to show that our dynamical system evolves according to a caricature of Coulomb interaction, which is not so obvious. We first go back to formulation (8), where $X$ should be considered as a time dependent square integrable map from $D$ into $\mathbf{R}^{d}$. We introduce

$$
\rho(t, x)=\int_{D} \delta(x-X(t, a)) d a,
$$

and we claim that, for each time $t$ such that $\rho(t,$.$) is absolutely continuous$ with respect to the Lebesgue measure, equation (8) is equivalent to :

$$
X^{\prime \prime}(t, a)=E(t, X(t, a)), \forall a \in D,
$$

where the acceleration field $E$ is given by

$$
E(t, x)=\frac{\nabla \Psi(t, x)-x}{\epsilon^{2}}
$$

and $\Psi$ is a solution of the Monge-Ampère equation

$$
\operatorname{det}\left(D^{2} \Psi(t, x)\right)=\rho(t, x),
$$

where $\operatorname{det}\left(D^{2} \Psi\right)$ stands for the determinant of the second derivatives of $\Psi(t, x)$ with respect to $x$. To justify this claim, we refer to the polar factorization theorem for maps (see $[\mathrm{Br}],[\mathrm{Ca}]$ ). At each time $t$ for which $\rho(t,$. is absolutely continuous with respect to the Lebesgue measure, we write (Theorem 1.2, p.377 in $[\mathrm{Br}]$ )

$$
X(t)=\nabla \Phi(t) \circ g(t)
$$

where $\Phi(t)$ is a function on $D$, with convex extension to the convex hull of $D$, and $g(t) \in S$ is a Lebesgue measure-preserving map from $D$ into itself. The factor $g(t)$ has additional properties (deduced from Theorem 1.2 and Proposition 2.2, p.390, in $[\mathrm{Br}])$. First, $g(t)$ is the unique point in $S$ that minimizes the $L^{2}$ distance to $X(t)$. Next, $X(t)-g(t)$ is the gradient of

$$
\left.X \in H \rightarrow \frac{1}{2} d_{H}^{2}(X, S)\right)
$$

at point $X=X(t)$. Finally, $g(t)$ can be written

$$
g(t)=\nabla \Psi(t) \circ X(t),
$$


where $\Psi$ (the Legendre-Fenchel transform of $\Phi$ with respect to $x \in D$ ) is a convex solution (in a suitable sense $[\mathrm{Br}],[\mathrm{Ca}]$ ) of the Monge-Ampère equation (13). So, we have obtained from the polar factorization theorem that

$$
\nabla_{X}\left(\frac{d_{H}^{2}(X(t), S)}{2}\right)=X(t)-\nabla \Psi(t) \circ X(t)
$$

and our claim is now justified.

So, we have obtained for the approximate geodesic equation (8) a MongeAmpère formulation with (10), (13), (11) and (12).

Now, as $\epsilon$ is small, a natural ansatz for $\Psi$ is,

$$
\Psi(t, x)=\frac{|x|^{2}}{2}-\epsilon^{2} \nabla \phi(t, x),
$$

which, inserted in (12) and (13), respectively leads to

$$
\begin{gathered}
E(t, x)=-\nabla \phi(t, x), \\
\rho(t, x)=1-\epsilon^{2} \Delta \phi(t, x)+O\left(\epsilon^{4}\right) .
\end{gathered}
$$

Dropping the $O\left(\epsilon^{4}\right)$ term in the last equation exactly gives the Poisson equation

$$
\rho(t, x)=1-\epsilon^{2} \Delta \phi(t, x),
$$

which involves the Coulomb potential. Since equations (10), (11), (18), (20) correctly describe a collisionless plasma of electrons with a uniform ion background and Coulomb interaction, we can say that our dynamical system of particles (which is an approximation as $h, \tau \rightarrow 0, \epsilon$ being fixed, of equation (8)) is just a caricature which gets finer as $\epsilon$ tends to zero. Let us finally observe that, in the very special case $d=1$, there is no discrepancy between the Monge-Ampère equation (13) and the Poisson equation (20) (because of (17)). Then, our dynamical system turns out to be an exact model of collisionless plasma, as shown in the Appendix.

\subsection{The semi-geostrophic equations}

Our dynamical system has an interesting connection with another physical model, namely Hoskins' frontogenesis model and the related semi-geostrophic equations in atmospheric sciences ([Ho], see also [CNP], $[\mathrm{BB}])$. A discrete version of this model has been discussed in $[\mathrm{BN}]$ and the corresponding particle system (in two dimensions) is given by

$$
i . X_{\alpha}^{\prime}(t)+X_{\alpha}(t)=A_{\sigma_{\alpha}(t)}
$$


where $i$ is the rotation matrix of angle $\pi / 2$ in $\mathbf{R}^{2}$ and $\sigma(t)$ is defined exactly in the same way as for our system of particles. In some vague sense, this Hamiltonian system has the same structural relationship with our system than the dynamical system for $N$ vortex points in $\mathbf{R}^{2}$ [MP] has with the dynamical system of $N$ electrons with Coulomb interaction in $\mathbf{R}^{d}$.

\section{Proof of the main result}

\section{$4.1 \quad$ Notations}

If $Y \in\left(\mathbf{R}^{d}\right)^{N}, v(t, Y)$ stands for $\left(v\left(t, Y_{\alpha}\right)\right)_{\alpha=1}^{N}$ and notations $v(t, X(t))$, $v(t, \sigma(t) A)$ etc...will be used. Partial derivatives in $\partial_{t} f$ and $\partial_{x_{i}} f$ are denoted by $f_{, t}$ and $f_{, i}$. There will be automatic summation on repeated latin indices $i, j$ and notation

$$
\sum_{\alpha}^{*}=\frac{1}{N} \sum_{\alpha}
$$

will be used. When possible, the explicit dependence on $t$ of $X$ and $\sigma$ will be omitted and capital letter will be used for functions of $X$, such as $V$ pour $v(t, X)$. For instance $\sum_{\alpha}^{*} X_{\alpha j} V_{\alpha i, j}$ means

$$
\frac{1}{N} \sum_{\alpha=1}^{N} \sum_{j=1}^{d}\left(X_{\alpha}\right)_{j}(t)\left(\partial_{x_{j}} v_{i}\right)\left(t, X_{\alpha}(t)\right) .
$$

\subsection{Bounds}

According to definition (2) and assumption (5), we have

$$
2 E(0)=\|v(t=0, A)\|^{2} \leq C .
$$

Since the total energy is non increasing, we deduce

$$
\left\|X^{\prime}(t)\right\| \leq C, \quad\|X(t)-\sigma(t) A\| \leq C \epsilon, \quad\|X(t)\| \leq C .
$$

\subsection{Modulated energy}

Let us introduce

$$
E_{\imath}(t)=\frac{1}{2}\left\|X^{\prime}(t)-v(t, X(t))\right\|^{2}+\frac{1}{2 \epsilon^{2}}\|X(t)-\sigma(t) A\|^{2},
$$


which can be seen as a modulated energy depending on $v$. Let us compute its time derivative on each interval $n \tau<t<(n+1) \tau$, where we know that the total energy $E(t)$ is preserved. We find

$$
\frac{d}{d t} E_{\nu}(t)=\sum_{\alpha}^{*}\left(-X_{\alpha i}^{\prime \prime} V_{\alpha i}+\left(V_{\alpha i}-X_{\alpha i}^{\prime}\right)\left(V_{\alpha i, t}+V_{\alpha i, j} X_{\alpha j}^{\prime}\right)\right)
$$

from which we get, using (1),

$$
\begin{gathered}
\frac{d}{d t} E_{\imath}(t)+\sum_{\alpha}^{*} V_{\alpha i, j}\left(V_{\alpha i}-X_{\alpha i}^{\prime}\right)\left(V_{\alpha j}-X_{\alpha j}^{\prime}\right) \\
=\sum_{\alpha}^{*}\left(V_{\alpha i}-X_{\alpha i}^{\prime}\right)\left(V_{\alpha i, t}+V_{\alpha j} V_{\alpha i, j}\right)+V_{\alpha i} \frac{X_{\alpha i}-\sigma A_{\alpha i}}{\epsilon^{2}}
\end{gathered}
$$

Rearranging (25), we obtain

$$
\frac{d}{d t} E_{\imath}(t)+Q(t)=I_{1}+I_{2}
$$

where $Q$ is defined by

$$
\sum_{\alpha}^{*} V_{\alpha i, j}\left(V_{\alpha i}-X_{\alpha i}^{\prime}\right)\left(V_{\alpha j}-X_{\alpha j}^{\prime}\right)-\frac{X_{\alpha i}-\sigma A_{\alpha i}}{\epsilon^{2}}\left(v_{i}\left(t, X_{\alpha}\right)-v_{i}\left(t, \sigma A_{\alpha}\right)\right)
$$

and $I_{1}, I_{2}$ by

$$
\begin{gathered}
I_{1}=\sum_{\alpha}^{*} \epsilon^{-2}\left(X_{\alpha i}-\sigma A_{\alpha i}\right) v_{i}\left(t, \sigma A_{\alpha}\right), \\
I_{2}=\sum_{\alpha}^{*}\left(V_{\alpha i}-X_{\alpha i}^{\prime}\right)\left(V_{\alpha i, t}+V_{\alpha j} V_{\alpha i, j}\right) .
\end{gathered}
$$

¿From the Euler equations (4), we get

$$
V_{\alpha i, t}+V_{\alpha j} V_{\alpha i, j}=-\left(p_{, i}\right)\left(t, X_{\alpha}\right)
$$

After setting

$$
D_{t} p=p_{, t}+v_{i} p_{, i}
$$

we see that

$$
\left(D_{t} p\right)\left(t, X_{\alpha}\right)=\frac{d}{d t}\left(p\left(t, X_{\alpha}\right)\right)+\left(p_{, i}\right)\left(t, X_{\alpha}\right)\left(V_{\alpha i}-X_{\alpha i}^{\prime}\right) .
$$


Thus $I_{2}$ becomes

$$
I_{2}=-\frac{d}{d t} J(t)+I_{3}+I_{4}
$$

where

$$
\begin{gathered}
J(t)=-\sum_{\alpha}^{*} p\left(t, X_{\alpha}(t)\right), \\
I_{3}=-\sum_{\alpha}^{*}\left(D_{t} p\right)\left(t, A_{\alpha}\right), \\
I_{4}=\sum_{\alpha}^{*}\left(D_{t} p\right)\left(t, A_{\alpha}\right)-\left(D_{t} p\right)\left(t, X_{\alpha}\right)
\end{gathered}
$$

which is also

$$
I_{4}=\sum_{\alpha}^{*}\left(D_{t} p\right)\left(t, \sigma A_{\alpha}\right)-\left(D_{t} p\right)\left(t, X_{\alpha}\right)
$$

We split $I_{1}=I_{5}+I_{6}$ with

$$
\begin{gathered}
I_{5}=-\sum_{\alpha}^{*} \epsilon^{-2} A_{\alpha i} V_{\alpha i}, \\
I_{6}=\sum_{\alpha}^{*} \epsilon^{-2} X_{\alpha i} v_{i}\left(t, \sigma A_{\alpha}\right)
\end{gathered}
$$

and (26) becomes

$$
\frac{d}{d t}\left(E_{v}(t)+J(t)\right)+Q(t)=I_{3}+I_{4}+I_{5}+I_{6}
$$

Since $v$ is smooth, by definitions (23) and (27), we have

$$
-Q \leq C E_{\imath}(t)
$$

and therefore (38) implies

$$
\frac{d}{d t}\left(E_{v}+J\right) \leq C E_{v}+I_{3}+I_{4}+I_{5}+I_{6}
$$

on each time interval $n \tau<t<(n+1) \tau$. Since $E(t)$ is preserved on each of these intervals with non-positive jumps at each $t=n \tau$, we deduce from definition (23) that $E_{\imath}(t)$ have the same jumps at $t=n \tau$ as $E(t)$ and, therefore, (40) is valid for all $0<t<T$ (in the sense of distributions). 


\subsection{Error estimates}

Let us first observe that, because of the assumption on the location of the $A_{\alpha}$, we have, for all Lipschitz continuous function $f$

$$
\left|\frac{1}{N} \sum_{\alpha} f\left(A_{\alpha}\right)-\int_{D} f(x) d x\right| \leq C \operatorname{Lip}(f) h .
$$

(Indeed, each $A_{\alpha}$ is assumed to belong to a $D_{\alpha}$, where each $D_{\alpha}$ has volume $N^{-1}$ and diameter no larger than $C h$.)

We first consider $I_{3}+I_{5}$, defined by (34), (36), that can be seen as a "quadrature formula" for the integral

$$
-\int_{D}\left(\left(\partial_{t}+v(t, x) \cdot \nabla\right) p(t, x)+\epsilon^{-2} x \cdot v(t, x)\right) d x
$$

Since $v$ is divergence-free and parallel to $\partial D$, this integral is simply

$$
\frac{d}{d t} \int p(t, x) d x
$$

But, the pressure can be normalized so that

$$
\int_{D} p(t, x) d x=0
$$

at each time $t$ and

$$
\left|I_{3}+I_{5}\right| \leq C h \epsilon^{-2}
$$

follows from (41). We immediately obtain for $I_{4}$, defined by (35),

$$
I_{4} \leq C\|X-\sigma A\| \leq C \epsilon E_{v}^{1 / 2} \leq \frac{1}{2} E_{\imath}+C \epsilon^{2} .
$$

To deal with $J$, defined by (33), we observe on one hand that

$$
\left|J+\sum_{\alpha}^{*} p\left(t, \sigma A_{\alpha}\right)\right| \leq C\|X-\sigma A\| \leq \frac{1}{2} E_{v}+C \epsilon^{2} .
$$

and on the other hand that

$$
\sum_{\alpha}^{*} p\left(t, \sigma A_{\alpha}\right)=\sum_{\alpha}^{*} p\left(t, A_{\alpha}\right)
$$

is a quadrature formula for $\int_{D} p(t, x) d x$ which is null by (44). Therefore

$$
|J(t)| \leq \frac{1}{2} E_{\imath}(t)+C\left(\epsilon^{2}+h\right) .
$$


Moreover, at $t=0$,

$$
J(0)=-\sum_{\alpha}^{*} p\left(t, A_{\alpha}\right)
$$

is a quadrature for $\int_{D} p(t, x) d x$ and

$$
|J(0)| \leq C h,
$$

follows from (44) and (41). Let us finally consider $I_{6}$, the most interesting term, defined by (37). Let us introduce

$$
I_{7}=\sum_{\alpha}^{*} \epsilon^{-2} X_{\alpha i}\left(t_{\tau}\right) v_{i}\left(t, \sigma\left(t_{\tau}\right) A_{\alpha}\right)
$$

where where $t_{\tau}$ stands for the integer part of $t / \tau$, multiplied by $\tau$. Thus,

$$
I_{7}-I_{6}=\sum_{\alpha}^{*} \epsilon^{-2}\left(X_{\alpha i}\left(t_{\tau}\right)-X_{\alpha i}(t)\right) v_{i}\left(t, \sigma\left(t_{\tau}\right) A_{\alpha}\right),
$$

where the dependence in $t$ and $t_{\tau}$ is explicitly written.

We immediately get that $I_{8}=I_{7}-I_{6}$ satisfies

$$
I_{8} \leq C \epsilon^{-2}\left|t-t_{\tau}\right| \sup _{0 \leq \theta \leq T}\left\|X^{\prime}(\theta)\right\|
$$

and, therefore,

$$
\left|I_{6}-I_{7}\right| \leq C \epsilon^{-2} \tau
$$

follows from (22). Let us introduce an artificial time step $\theta>0$ so that

$$
\sup _{a \in D}\left|v(t, a)-\frac{M(t, t+\theta, a)-a}{\theta}\right| \leq C \theta
$$

where we denote by $M\left(t_{0}, t_{1}, a\right)$ the location in $D$ at time $t_{1}$ of a point advected by the velocity field $v(t, x)$ and located at $a$ at time $t_{0}$. Let us introduce

$$
I_{9}=\sum_{\alpha}^{*} \epsilon^{-2} X_{\alpha i}\left(t_{\tau}\right) \frac{M_{i}\left(t, t+\theta, \sigma\left(t_{\tau}\right) A_{\alpha}\right)-\sigma\left(t_{\tau}\right) A_{\alpha i}}{\theta}
$$

which is an approximation of $I_{7}$, defined by $(50)$. Indeed,

$$
\left|I_{7}-I_{9}\right| \leq C \theta \epsilon^{-2}|| X\left(t_{\tau}\right)|| \leq C \theta \epsilon^{-2}
$$

follows from (53) and (22). Since $v$ is a smooth divergence-free vector field, parallel to $\partial D$, the mapping $a \rightarrow M(t, t+\theta,$.$) is a Lebesgue measure-$ preserving Lipschitz transformation of $D$. Following a result of Lax [La], we 
observe that such a transformation can be approximated, in sup norm with an error of order $h$, by a permutation of the $A_{\alpha}$. More precisely, there exists a permutation $\eta$ such that

$$
\sup _{\alpha=1, \ldots, N}\left|M\left(t, t+\theta, A_{\alpha}\right)-\eta A_{\alpha}\right| \leq C h .
$$

Thus

$$
\left|I_{9}-I_{10}\right| \leq C \frac{h}{\theta} \epsilon^{-2}
$$

where

$$
I_{10}=\sum_{\alpha}^{*} \epsilon^{-2} X_{\alpha i}\left(t_{\tau}\right) \frac{\eta A_{\alpha i}-\sigma\left(t_{\tau}\right) A_{\alpha i}}{\theta}
$$

This last expression is always non-positive, since, by construction $(3), \sigma\left(t_{\tau}\right)$ satisfies

$$
\left\|X\left(t_{\tau}\right)-\sigma\left(t_{\tau}\right) A_{\alpha}\right\|^{2} \leq\left\|X\left(t_{\tau}\right)-\eta A_{\alpha}\right\|^{2} .
$$

So, from (52), (55), (57) we deduce

$$
I_{6} \leq C\left(\frac{h}{\theta}+\theta+\tau\right) \epsilon^{-2} .
$$

Using (47), (45), (46) and (59) in the right-hand side of (40), we get

$$
\frac{d}{d t}\left(E_{\nu}+J\right) \leq C\left(E_{v}+J\right)+C\left(\frac{h}{\theta}+\theta+\tau\right) \epsilon^{-2}+C\left(\epsilon^{2}+h\right) .
$$

By setting the artificial parameter $\theta$ equal to $h^{1 / 2}$ and using assumption (6), we get

$$
\left(E_{v}+J\right)(t) \leq C\left(E_{v}+J\right)(0)+C \epsilon^{2} .
$$

Since $E_{\imath}(0)=0$ follows from assumption (5), we deduce from (47) and (49) that

$$
E_{\nu}(t) \leq C \epsilon^{2},
$$

which concludes the proof, by definition (23).

\section{Appendix : the one-dimensional case}

As already mentioned in subsection 3.2, we can expect, in the special case $d=1$, that our dynamical system is an exact description of a collisionless plasma of electrons with a fixed and uniform ion background (see [BR], [ZM], [MMZ], for some mathematical and numerical aspects of this model). To check this statement, let us consider a uniform background of non-moving 
ions in $\mathbf{R}^{3}$ and $N$ layers of electrons, all of them being orthogonal to a fixed axis. Each layer can be considered as a particle moving along this axis, with position $X_{\alpha}(t) \in \mathbf{R}$ at time $t$, for $\alpha=1, \ldots, N$. The electric field is scalar, depends on one space variable $x \in \mathbf{R}$ and satisfies

$$
\partial_{x} E(t, x)=1-\frac{1}{N} \sum_{\alpha} \delta\left(x-X_{\alpha}(t)\right)
$$

Thus we get for each particle

$$
X_{\alpha}^{\prime \prime}(t)+X_{\alpha}(t)=\frac{1}{N} \sum_{\beta=1}^{N} H\left(X_{\alpha}(t)-X_{\beta}(t)\right)-E_{0}(t),
$$

where $H$ stands for the Heaviside function, with conventionally $H(0)=1 / 2$, and $E_{0}(t)$ depends on the boundary conditions we choose. Let us now consider each time $t$ when all particles have different locations, sort their positions by increasing order and denote by $\sigma_{\alpha}(t)$ the rank of the particle with label $\alpha$, for $\alpha=1, \ldots, N$. This defines a (time-dependent) permutation $\sigma=\sigma(t)$ that can be easily seen as the one that minimizes

$$
\sum_{\alpha}\left|X_{\alpha}(t)-A_{\sigma_{\alpha}}\right|^{2}
$$

where $A_{\alpha}=(\alpha-1 / 2) / N$, for $\alpha=1, \ldots, N$. Thus (62) becomes

$$
X_{\alpha}^{\prime \prime}(t)+X_{\alpha}(t)=A_{\sigma_{\alpha}(t)}-E_{0}(t)
$$

So, up to the choice of suitable boundary conditions to enforce $E_{0}(t)=0$, we have recovered our system of particles (up to the further time discretization

with time-step $\tau>0$ ) with $D=[0,1]$ and the fictitious 'ions' $A_{\alpha}$ standing for the ion background.

\section{Acknowledgments}

The author thanks the Erwin Schrödinger Institute (ESI), the University of Toronto and the Courant Institute where this work has been completed. This work has also been supported by TMR "asymptotic methods in kinetic equations" and the program on Charged Particle Kinetics at the ESI. 


\section{References}

[AK] V.I.Arnold, B.Khesin, Topological methods in hydrodynamics, Springer, New York, 1998.

[BN] S. Baigent, J. Norbury, Two discrete models for semi-geostrophic dynamics, Phys. D 109 (1997) 333-342.

[BR] J.Batt, G.Rein, Global classical solutions of the periodic VlasovPoisson system, C.R. Acad. Sci. Paris 313 (1991) 411-416.

[BB] J.-D. Benamou, Y. Brenier, Weak existence for the semigeostrophic equations formulated as a coupled Monge-Ampère/transport problem, SIAM J. Appl. Math. 58 (1998) 1450-1461.

[Br] Y. Brenier, Polar factorization and monotone rearrangement of vectorvalued functions, Comm. Pure Appl. Math. 44 (1991) 375-417.

[Br2] Y.Brenier, Minimal geodesics on groups of volume-preserving maps, Comm. Pure Appl. Math. 52 (1999) 411-452.

[Eb] D. Ebin, The motion of slightly compressible fluids viewed as a motion with strong constraining force, Ann. of Math. (2) 105 (1977) 141-200.

[Ca] L. Caffarelli, Boundary regularity of maps with convex potentials, Comm. Pure Appl. Math. 45 (1992) 1141-1151.

[CNP] M. Cullen, J. Norbury, J. Purser, Generalised Lagrangian solutions for atmospheric and oceanic flows, SIAM J. Appl. Math. 51 (1991), $20-31$.

[Ho] B. Hoskins, The mathematical theory of frontogenesis, Annual review of fluid mechanics, Vol. 14, pp. 131-151, Palo Alto, 1982.

[La] P. Lax, Approximation of measure preserving transformations, Comm. Pure Appl. Math. 24 (1971) 133-135.

[MMZ] A. Majda, G. Majda, Y.X. Zheng, Concentrations in the onedimensional Vlasov-Poisson equations. I. Temporal development and non-unique weak solutions in the single component case, Phys. D 74 (1994) 268-300.

[MP] C.Marchioro, M.Pulvirenti, Mathematical theory of incompressible nonviscous fluids, Springer, New York, 1994. 
[RU] H. Rubin, P. Ungar, Motion under a strong constraining force, Comm. Pure Appl. Math. 10 (1957) 65-87.

[Sh] A. I. Shnirelman, Generalized fluid flows, their approximation and applications, Geom. Funct. Anal. 4 (1994) 586-620.

[ZM] Y.X. Zheng, A. Majda, Existence of global weak solutions to onecomponent Vlasov-Poisson and Fokker-Planck-Poisson systems in one space dimension with measures as initial data, Comm. Pure Appl. Math. 47 (1994) 1365-1401. 